\title{
Fast Method for Computing Chemical Potentials and Liquid-Liquid Phase Equilibria of Macromolecular Solutions
}

Sanbo Qin and Huan-Xiang Zhou*

Supplementary Figures and Tables

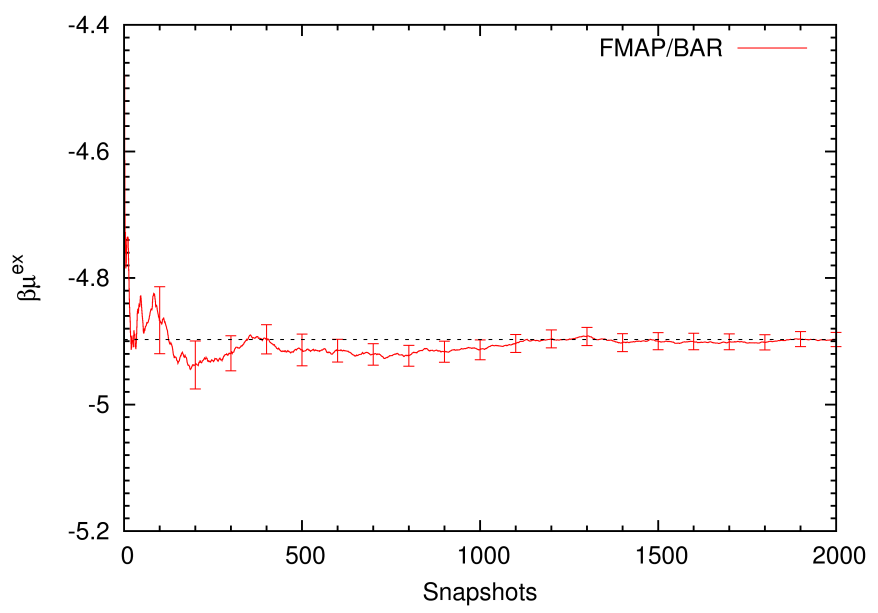

Figure S1. Evolution of the excess chemical potential over Monte Carlo simulation steps. Snapshots are collected every 10,000 steps. Results shown are for $T=0.65$ and $N=360$, calculated by the FMAP/BAR method. Errors were estimated by Flyvbjerg and Petersen's block decorrelation technique, with blocks consisting of fixed-length stretches of snapshots. The estimated errors are almost flat over various block sizes, indicating that successive snapshots are separated by a sufficient number of simulation steps as to produce little correlation within our data. 


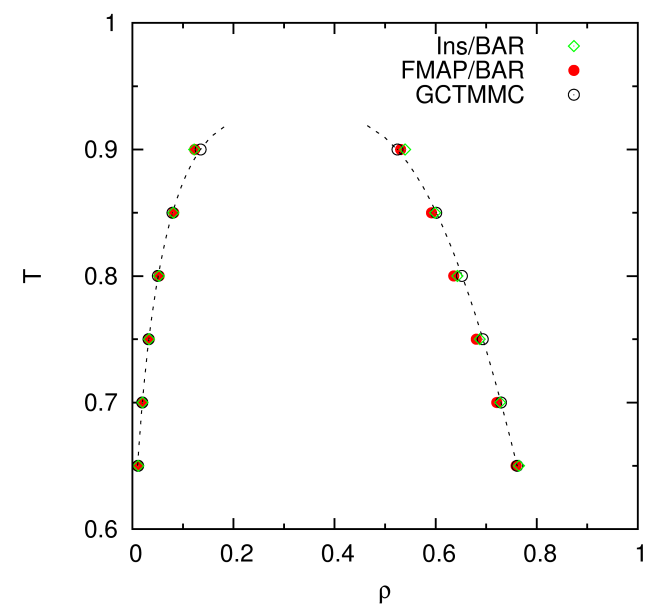

Figure S2. Gas-liquid coexistence densities at different subcritical temperatures calculated by Ins/BAR and FMAP/BAR, compared to previous GCTMMC results. The results by brute-force insertion (Ins) and FMAP-enabled insertion have good agreement at all temperatures, indicating that the correction for FMAP discretization error is satisfactory. These results show growing, though still modest, discrepancy from the GCTMMC benchmark, at higher temperatures, attributable in part to system size effects.

Table S1. The first-order coefficient $b_{1}$ of the polynomial fit of $\mu^{\text {ex }}$ and its theoretical expected value $2 B_{2}$.

\begin{tabular}{cccc}
\hline$T$ & $b_{1}$ & $2 B_{2}$ & $b_{1} / 2 B_{2}$ \\
\hline 0.65 & -18.3 & -15.2 & 1.20 \\
0.70 & -16.1 & -13.2 & 1.22 \\
0.75 & -13.6 & -11.5 & 1.18 \\
0.80 & -11.9 & -10.2 & 1.15 \\
0.85 & -10.3 & -9.0 & 1.08 \\
0.90 & -8.7 & -8.1 & \\
\hline
\end{tabular}

